\title{
Dendritic cells in intestinal homeostasis and disease
}

\author{
Maria Rescigno' ${ }^{1}$ and Antonio Di Sabatino² \\ ${ }^{1}$ Department of Experimental Oncology, European Institute of Oncology, Milan, Italy. \\ ${ }^{2}$ First Department of Medicine, S. Matteo Hospital, University of Pavia, Pavia, Italy.
}

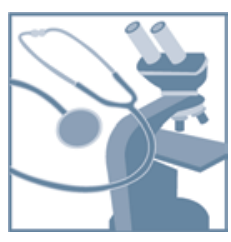

\begin{abstract}
DCs are specialized APCs that orchestrate innate and adaptive immune responses. The intestinal mucosa contains numerous DCs, which induce either protective immunity to infectious agents or tolerance to innocuous antigens, including food and commensal bacteria. Several subsets of mucosal DCs have been described that display unique functions, dictated in part by the local microenvironment. In this review, we summarize the distinct subtypes of DCs and their distribution in the gut; examine how DC dysfunction contributes to intestinal disease development, including inflammatory bowel disease and celiac disease; and discuss manipulation of DCs for therapy.
\end{abstract}

DCs migrate from peripheral tissues to secondary lymphoid organs, where they present antigens to T cells (1). Although initially considered only as activators of the immune response to external insults (including infectious agents and transformed cells), recent reports showed that DCs play a major role in the maintenance and initiation of immune tolerance (2). Tolerance is a process that prevents the induction of immunity to innocuous antigens, be they self or non-self antigens (i.e., those introduced as part of an individual's diet; ref. 3). In the intestine, tolerance is also induced toward commensal microorganisms that are required for digestive function (3).

Circulating DC precursors seed peripheral tissues and lymphoid organs, where they differentiate into either plasmacytoid or conventional DCs that display different functions (4). DCs are found in 2 functionally distinct states: immature and mature cells. Immature DCs are present in peripheral tissues and are mainly phagocytic cells; mature DCs are found in lymphoid organs and are specialized in antigen presentation, as they are characterized by the high surface expression of costimulatory molecules that are required for $\mathrm{T}$ cell activation (1). Mature DCs derive from immature cells after a maturation process that is initiated by inflammatory stimuli and that leads to a massive migration of DCs to draining lymph nodes. However, there is a low but constant migration of immature/semimature DCs from peripheral tissues to draining lymph nodes (5). These cells are likely involved in tolerance induction to self antigens (2).

Particularly in the gut, a subset of DCs (described in detail below) has been shown to preferentially drive the development of Tregs, the principal effectors of tolerance (6). CD4 ${ }^{+} \mathrm{T}$ cells are subdivided into T helper cells (Th1, Th2, and Th17 cells) and Tregs. Th1 cells are inflammatory cells that release IFN- $\gamma$ and are involved in immunity against intracellular pathogens, whereas Th2 cells are primarily involved in B cell help as they release B cell growth factors like IL-4. Th17 cells play a critical role in host defense against a variety of bacteria and fungi (7), but under pathologic conditions such as autoimmunity, Th17 cells exacerbate inflammation (8). Tregs suppress the function of effector $\mathrm{T}$ cells and are thus essential to counteract inflammatory responses (9). The capacity

Conflict of interest: The authors have declared that no conflict of interest exists. Citation for this article: J. Clin. Invest. 119:2441-2450 (2009). doi:10.1172/JCI39134. of intestinal DCs to induce Tregs is only one of their specialized functions (6). Intestinal DCs actively participate in antigen capture across the intestinal epithelium by extending protrusions directly into the lumen for antigen sampling (10). Intestinal DCs also impart gut-homing properties to lymphocytes (reviewed in ref. 6) or induce class switch recombination to IgA (the process by which B cells acquire expression of the most abundant antibody in mucosal secretions, IgA, which aids several mucosal functions, including immune-mediated exclusion of both pathogenic and commensal microorganisms; ref. 11).

Given the important role played by DCs in intestinal homeostasis, in this review we describe recent advances in our knowledge of intestinal DC functions and how dysregulation of these functions contributes to intestinal diseases. We also discuss the potential of manipulation of DCs for therapeutic purposes.

\section{DC distribution in the gut}

DCs are found throughout the intestine, including the lamina propria (LP) of the small and large intestine, the isolated lymphoid follicles, the Peyer patches (PPs), and the mesenteric lymph nodes (MLNs; recently reviewed in refs. 6, 12). PPs are organized lymphoid tissues responsible primarily for the induction of mucosal $\mathrm{T}$ cell-dependent IgA responses. In mouse PPs, DCs are found both in the subepithelial dome and in the interfollicular $T$ cell regions (13). Several subsets of DCs have been described in the PPs as having distinct functions (Table 1). The LP of the small intestine is home to a higher frequency of DCs (11\%) than is the spleen $(3 \%)$ in C57BL/6 mice (14). Although many different LP DC subsets have been described in the mouse (Table 1), functionally, LP DCs are divided into 2 major classes according to their ability to differentially activate lymphocytes. These $2 \mathrm{DC}$ types are distinguished by the expression of CD103, the receptor for the epithelial cell adhesion molecule E-cadherin $(6,15)$. CD $103^{+}$DCs are also found in the MLNs, but not in the spleen $(14,16,17)$, and likely represent a population of DCs migrating from the LP, as they are reduced in number in $\mathrm{Ccr}^{-/-}$mice (18). Furthermore, MLN CD $103^{+}$DCs incorporate BrdU with kinetics slower than those of MLN CD103DCs or LP CD $103^{+}$DCs, which suggests that CD $103^{+}$DCs acquire BrdU in the LP and then migrate to the MLN (19).

Human intestinal DCs are poorly characterized. These cells migrate out from colon biopsies after overnight culture and dis- 


\section{Table 1}

DC distribution and function in the intestine

\begin{tabular}{|c|c|c|c|}
\hline DC subset & Localization & Function & Reference \\
\hline \multicolumn{4}{|l|}{ PP } \\
\hline CX3CR1+ & SED & Unknown & (126) \\
\hline $\mathrm{CCR}^{+}$ & SED & Th1-polarizing ability & $(126)$ \\
\hline $\mathrm{CD} 11 \mathrm{~b}^{+}$ & SED & Th2-polarizing ability; IgA class switching & $(33-35)$ \\
\hline $\mathrm{CD}^{+}$ & IFR & Th1-polarizing ability & $(33)$ \\
\hline CD11b-CD8-B220- & SED & Th1-polarizing ability & (33) \\
\hline$B 220+L y 6 C+(p D C)$ & SED, IFR & Reduced type I IFN production & (53) \\
\hline \multicolumn{4}{|l|}{ Small intestine } \\
\hline CD11chiCD11b hi/locD103+ & LP & Treg-polarizing ability & $(14)$ \\
\hline CD11 $\mathrm{c}^{\mathrm{h} / / /} \mathrm{CX} 3 \mathrm{CR} 1+C D 70+C D 11 \mathrm{~b}^{\mathrm{hi}} \mathrm{CD} 103^{-}$ & Intraepithelial & Th17-polarizing ability & $(15,25)$ \\
\hline  & Intraepithelial? & Th17-polarizing ability; IgA class switching & $(24)$ \\
\hline 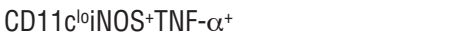 & LP & IgA class switching & (36) \\
\hline \multicolumn{4}{|l|}{ MLN } \\
\hline CD11chiCD103+ & & Treg-polarizing ability; gut-homing T cell imprinting & $(17-19)$ \\
\hline CD11 $^{\text {hi CD103- }}$ & & Th stimulatory & $(17)$ \\
\hline $\mathrm{CD}_{11 c^{\text {hic }}} \mathrm{COX}^{+}$ & & Oral tolerance & $(127)$ \\
\hline
\end{tabular}

IFR, interfollicular region; pDC, plasmacytoid DC; SED, subepithelial dome.

play a potent immunostimulatory capacity (20); however, they fail to activate Th 1 cells, as they are unable to release IL-12 in response to inflammatory stimuli (21). A population of $\mathrm{CD} 103^{+} \mathrm{DCs}$ has also been described in human MLNs (19).

\section{Unique properties of mucosal DCs}

Tcell activation. As mentioned above, DCs isolated from intestinal tissues are endowed with unique mucosal functions. These include the ability to drive the de novo conversion of immunoregulatory Foxp $3^{+}$Tregs, the differentiation of Th17 cells, and the promotion of IgA class switching. These tasks are specific for certain DC subsets (Table 1). CD $103^{+}$DCs isolated either from the LP or from the MLNs promote the differentiation of Foxp $3^{+}$Tregs (Figure 1 and refs. 14, 17). This activity is dependent on retinoic acid (RA; a metabolite of vitamin A degradation) and TGF- $\beta(14,17,22)$. TGF- $\beta$ plays an important role in the homeostasis of the gut (23). LP CD $103^{+}$DCs express high levels of CD11c, but they do not express CX3CR1 $(14,15,24,25)$. This is interesting because DCs extending dendrites into the lumen are CX3CR1 ${ }^{+}(26)$, suggesting that these cells may not be involved in tolerance induction. Despite the clear ability of gut CD $103^{+}$DCs to activate Tregs, DCs isolated from the cannulated lymph draining the intestine of rats are strongly immunogenic, which suggests that outside their local microenvironment, DCs may lose their unique phenotype, even though an inflammatory event associated with cannulation cannot be excluded (27). RA has been shown to inhibit the development of Th17 cells (22), making it unlikely that CD103+ DCs polarize both Tregs and Th17 cells. Indeed, the differentiation of Th17 cells seems to depend on another LP DC subset that expresses high levels of CD11b and TLR5 (Figure 1 and refs. 24, 25). TLRs are receptors for microbe-associated molecular patterns and allow for sensing of microbes (28). The involvement of TLR5 is interesting, because LP DCs either express very few TLRs or are refractory to most TLR-mediated stimulation (29). Consequentially, LP DCs are less responsive to microbial stimulation, but may still respond to flagellated motile bacteria, as flagellin is the ligand of TLR5 (Figure 1).
A second population of LP DCs promoting Th17 differentiation is characterized by the expression of CD70 and CX3CR1 (15). This population also expresses a series of ATP receptors and induces vigorous Th17 cell differentiation from naive T cells in the presence of ATP, but independently of TLR signaling (15). Th17 cells are absent in mice reared under germ-free conditions $(15,30)$, but this finding remains controversial (31). The administration of ATP restores Th17 cell differentiation in germ-free mice, which suggests that bacteria-derived ATP is responsible for Th17 cell differentiation via the action of CX3CR $1^{+} \mathrm{CD} 70^{+} \mathrm{DCs}(15)$. It is not clear whether these cells are somehow related to $\mathrm{CD} 70^{+} \mathrm{APCs}$ of non-bone marrow origin that are required for $\mathrm{T}$ cell proliferation directly in the LP (32). Surface markers expressed by these 2 cell types would argue against this possibility.

$B$ cell activation. The $\mathrm{CD}_{1} 1 \mathrm{~b}^{+}$subset of PP DCs releases IL-10, TGF- $\beta$ (33), IL-6, and RA, which are all required for isotype switching to IgA in PPs $(34,35)$. T cell-dependent IgA class switching is regulated by TNF- $\alpha /$ iNOS-producing PP DCs (36) that express the integrin $\alpha_{v} \beta_{8}$ involved in TGF- $\beta$ activation (37). However, DCs also drive the development of IgA directly in the isolated lymphoid follicles of the LP via a primitive T cell-independent pathway (38, 39). The same subset of LP DCs driving Th17 cell development is also responsible for IgA induction after triggering via TLR5 in an RA-dependent manner in the mouse (24). An additional LP DC subset expressing TNF- $\alpha$ and iNOS has also been shown to favor IgA class switching (Table 1$)$.

\section{DC interaction with the environment}

In the small intestine, DCs have been shown to differentiate from circulating inflammatory monocytes after depletion of resident DCs and macrophages (40). Once inside the tissue, DC precursors acquire mucosal functions via interaction with the local environment. These cells differentiate into distinct subsets with specialized functions based on the type of DC precursor, which explains why subsets of DCs isolated from different organs display distinct phenotypes. Here, we focus on 3 cell types that have been recently reported to release factors that influence DC function. 


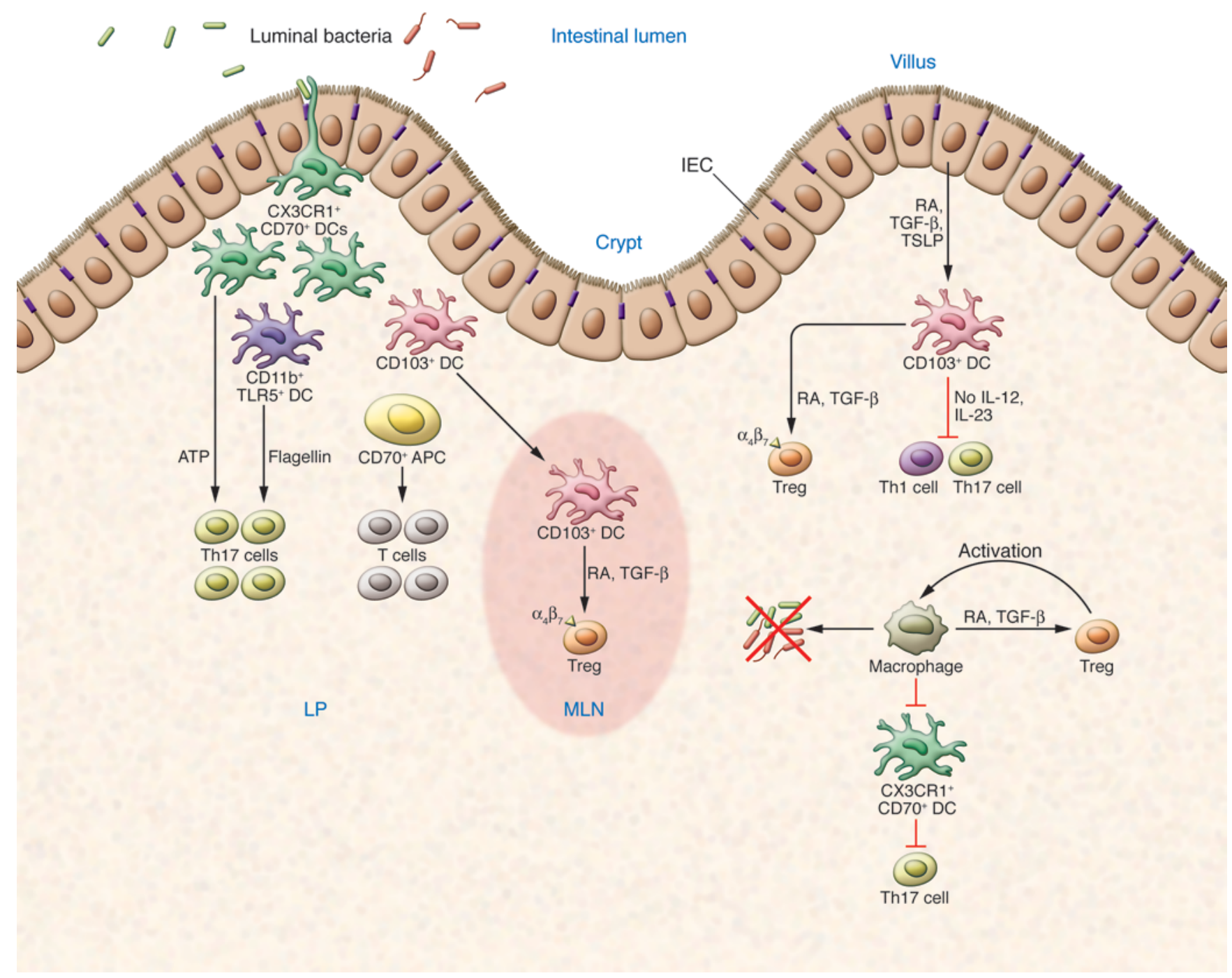

Figure 1

DC distribution and function in the LP. Left: CX3CR1+ DCs extend protrusions across the epithelial barrier. These cells also express CD70 and drive the differentiation of Th17 cells via a mechanism dependent on ATP and/or flagellin. CD103+ DCs migrate into the draining MLN, where they promote the conversion of Foxp3 ${ }^{+}$Tregs via an RA- and TGF- $\beta$-dependent mechanism. Tregs also upregulate the expression of the gut-homing marker $\alpha_{4} \beta_{7}$. A third population of APCs of non-bone marrow origin expressing CD70+ is required for T cell proliferation directly in the LP. Right: The phenotype of CD103+ LP DCs is conferred by the local microenvironment, in particular by IECs via the release of TGF- $\beta$, RA, and - in the human system - TSLP. CD103+ DCs acquire the ability to drive the differentiation of Tregs and to inhibit Th1 and Th17 cell development. Macrophages also limit intestinal inflammation via activation of Tregs and inhibition of the ability of CX3CR1+ DCs to drive Th17 cell development. Macrophages retain full antibactericidal activity.

Epithelial cell-derived signals. Human intestinal epithelial cells (IECs) play an important role in driving the development of noninflammatory DCs (41). IECs release thymic stromal lymphopoietin (TSLP), which inhibits IL-12 production by DCs in response to bacteria and drives the development of Th2-polarizing DCs (21). In agreement with these findings, impairment of NF- $\mathrm{KB}$ signaling by IKK- $\beta$ deletion in IECs in mice reduces TSLP expression and upregulates DCderived IL-12p40 (42). This is associated with the inability to drive Th2 cell differentiation and to control infection with Trichuris muris whipworms (42). IECs also release TGF- $\beta$ and RA and are responsible for driving the development of $\mathrm{CD} 103^{+}$tolerogenic DCs from CD103- cells. These cells also inhibit Th1 and Th17 cell development, which indicates that IECs imprint a full mucosal phenotype on DCs (Figure 1 and ref. 43). In addition, TSLP favors the release of a proliferation-inducing ligand (APRIL) and B cell-activation factor of the TNF family (BAFF) by IEC-conditioned DCs and supports IgA class switching directly in the $\operatorname{LP}(44,45)$ in humans (39). APRIL and BAFF are CD40 ligand-related molecules required for IgA class switching $(11,46)$. Notably, incubation of bacteria with IECs results in different levels of TSLP and TGF- $\beta 1$ upregulation, depending on the nature of the encountered bacteria in vitro, with Gram-negative species being the most effective at inducing such upregulation (47). Together with the observation that bacteria-derived ATP shapes the response of DCs (15), the ability of bacteria to induce immunomodulatory mediator expression by IECs suggests that bacteria influence DC function both directly and indirectly via their action on IECs. 




\section{Figure 2}

Three non-mutually exclusive possible mechanisms of DC involvement in IBD that lead to an imbalance between Th17/Th1 and Treg cells have been reported. (A) Involvement of ATP-releasing or flagellated bacteria. An unexpected increase in the number of bacteria releasing ATP or expressing flagellin can lead to the activation of CX3CR1 ${ }^{+}$CD70+ DCs that favor Th17 cell differentiation (i). (B) Involvement of the local microenvironment. A defect in the release of immunomodulatory factors (e.g., TSLP, TGF- $\beta$, and RA) by IECs may lead to a reduction in Treg numbers caused by the failure of conditioning tolerogenic CD103+ DCs (ii). Local inflammation may lead to the recruitment of inflammatory DCs; by releasing IL-12 and TNF- $\alpha$, these inflammatory DCs drive the differentiation of IFN- $\gamma$ and TNF- $\alpha$ Th1 cells (iii). (C) Involvement of immune cells. Inflammation may also affect the differentiation of tolerogenic macrophages from recruited monocytes, leading to reduction in Treg differentiation and inability to control the activity of CX3CR $1{ }^{+} \mathrm{CD} 70^{+} \mathrm{DCs}$ (iv). Th17 or Th1 cells are strongly restimulated in situ by $\mathrm{CD} 70^{+}$or $\mathrm{OX} 40 \mathrm{~L}^{+} \mathrm{APCs}$ (v). Both DC types have not been described in humans, but the retention of activated DCs has been shown. The mechanisms in $\mathbf{A}-\mathbf{C}$ may participate in disease induction by generating an imbalance between Tregs and Th1 or Th17 cells (vi). Th1/Th17 cells release IFN- $\gamma$, TNF- $\alpha$, or IL-17, which contribute to tissue destruction through the release of MMPs by activated fibroblasts and the recruitment of neutrophils. Th1 or inflammatory, DC-derived TNF- $\alpha$ may also increase the endothelial expression of MAdCAM-1, thus favoring the recruitment of $\alpha_{4} \beta_{7}{ }^{+}$Th1 cells.

Stromal cell-derived signals. Stromal cells play an important role in driving the development of tolerogenic macrophages in the gut $(48)$ and regulatory DCs in the spleen $(49,50)$. Under the influence of stroma-derived TGF- $\beta$, intestinal macrophages - although perfectly capable of exerting bactericidal activity - are unable to release proinflammatory cytokines (48). Intestinal macrophages also drive the development of $\mathrm{CD} 4{ }^{+} \mathrm{CD} 25^{+} \mathrm{FoxP} 3^{+}$Tregs via a TGF- $\beta$ - and RAdependent mechanism (25). It would be interesting to know whether stromal cells, like IECs, express retinal dehydrogenase and are able to convert retinal into RA. In addition, stromal cells constitutively express COX2 and produce prostaglandin E2 (51), which affects the activity of human conventional DCs (52) and inhibits the production of type I IFNs in mouse plasmacytoid DCs (pDCs; ref. 53).

Immune cell-derived signals. The ability of intestinal macrophages to control DC function has recently been proposed (25). DCs in coculture with intestinal macrophages have a reduced capacity to induce Th17 cells, independent of IL-10 production (Figure 1 and ref. 25). In addition, LP macrophages express aldehyde dehydrogenase family 1 , subfamily $1 \mathrm{~A}$ (Aldh1a1) and Aldh1a2, which suggests that LP macrophages may be another source of RA to drive Treg 
differentiation. The exact nature of these macrophages remains to be established, as they also express CD103 (25). Furthermore, macrophages release GM-CSF, which has been shown to induce Aldh1a2 expression in bone marrow-derived DCs and to favor Treg development (54). Notably, Tregs steer monocyte differentiation toward antiinflammatory macrophages, which suggests that these cell types might control each other's development and function (55).

\section{DCs in intestinal disease}

Inflammatory bowel diseases (IBDs), which include Crohn disease and ulcerative colitis, are chronic relapsing inflammatory diseases of the gastrointestinal tract. The highest prevalence of Crohn disease is in Western countries, with average incidence ranging 100-200 cases per 100,000 (56). Although Crohn disease and ulcerative colitis share many symptoms (e.g., diarrhea, abdominal pain, anemia, and weight loss), they have a number of pathological differences. In Crohn disease, the ileum is the most frequent site of inflammation, but lesions may occur anywhere along the digestive tract from the mouth to the anus. In ulcerative colitis, the colon is typically the only affected site. Ulcerative colitis affects the sole mucosal layer, begins in the rectum, and spreads up through the colon, while in Crohn disease, the inflammation is transmural and patchy. Crohn disease is complicated by perianal fistulas, abscesses, and intestinal strictures leading to obstructions, while ulcerative colitis may predispose to colorectal cancer or evolve to toxic megacolon (57).

The immunopathology of these disorders relates to an inappropriate and exaggerated mucosal immune response to constituents of the gut flora in genetically predisposed individuals (58). The exact role of DCs in the etiology of IBD is unknown, but several observations in humans and in mouse models of IBD suggest that DCs may play a pathogenic role (Figure 2). As mentioned above, the function of mucosal DC subsets is tightly regulated by the local microenvironment, which includes immune cells, nonimmune cells, and luminal bacteria. All of these factors participate in preserving intestinal homeostasis. Hence, deregulation at 1 or more of these 3 levels may affect DC function and cause intestinal disease (Figure 2). Dysfunctional DCs may act by priming abnormal $\mathrm{T}$ cell responses to the enteric flora in organized lymphoid tissues, sustain $T$ cell reactivity within the inflamed mucosa through the interaction with $\mathrm{T}$ cells, and function as effector cells via the release of proinflammatory cytokines. Furthermore, there may be an imbalance between Th17 cell- versus Treg-inducing DC subsets, favoring Th17 cell differentiation and thus driving inflammation. In the following paragraphs, we analyze the role of DCs in intestinal disease with particular reference to experimental colitis in the mouse, IBD, and celiac disease.

Role of DCs in experimental models of colitis. A possible pathogenic role of DCs - either in the establishment or in the maintenance of colitis - has emerged in different mouse models of intestinal inflammation resembling IBD. In the $T$ cell transfer model of colitis, which involves the transfer of $\mathrm{CD} 45 \mathrm{RB}^{\mathrm{hi}} \mathrm{CD} 4^{+} \mathrm{T}$ cells from immunocompetent mice to SCID mice, a higher number of CD $11 c^{+}$DCs expressing the activation marker OX40 ligand (OX40L) were identified in the MLNs (59), and transplanted $\mathrm{T}$ cells formed aggregates with CD $11 \mathrm{c}^{+} \mathrm{DCs}$ in the LP (58). Blocking OX40-OX40L interactions prevented the induction of colitis (60).

DCs also play a pathogenic role in T cell-independent models of colitis. For instance, DC activation via the costimulatory molecule CD40 causes gut inflammation in the absence of B and T cells via the release of inflammatory cytokines, including IL-23 and IL-17 (61). Oral administration of dextran sulfate sodium (DSS) induces an acute form of colitis. Diphtheria toxin-induced (DTinduced) ablation of DCs in DT receptor-transgenic mice during DSS administration ameliorates colitis (62). When mice are pretreated with immunostimulatory DNA sequences before DSS administration, the presence of DCs is protective, partly because of type I IFN release that regulates the recruitment of neutrophils and monocytes and their inflammatory activities in the inflamed colon (62). Conversely, if DCs are ablated before DSS treatment, colitis is exacerbated (63), which suggests that DCs play a protective role in the initial phases of colitis but play a pathogenic role at a later time in disease course. There might be several mechanisms by which resident DCs could protect the colon during the initiation of colitis, but their ability to induce Treg development may play a primary role. For example, clusters of DCs and Tregs are described in the colonic mucosa of mice during amelioration of colitis (64), CD $103^{+}$DCs are required for the suppression of colitogenic T cells $(14,16,17)$, and mice lacking integrin $\alpha_{v} \beta_{8}$ on DCs have reduced numbers of Tregs in colonic tissue and develop colitis (37). In addition, Treg expansion appears to occur through antigen-specific enterocyte-T cell interactions (65), suggesting a further level of control exerted by IECs.

The involvement of DCs during the late phases of colitis development may be caused by aberrant activation of resident DCs, recruitment of DCs that were not exposed to the local tolerogenic microenvironment and hence are immunogenic, and an imbalance between tolerogenic and immunogenic DCs. Analysis of the DC phenotype in murine colitis has shown an expansion of mature DCs expressing higher levels of costimulatory molecules (CD40, CD80, and CD86) and increased amounts of IL-12p40 and IL-23p19 upon CD40 ligation (66). Together, IL-12p40 and IL-23p19 form IL-23, which is important for the growth and stabilization of Th17 cells in the mouse and their differentiation in humans (67). In a transgenic mouse expressing firefly luciferase under control of the $I L-12 p 40$ promoter, it was shown that CD8 $\alpha^{-} \mathrm{CD} 11 \mathrm{~b}^{-} \mathrm{CD} 11 \mathrm{c}^{+} \mathrm{DCs}$ located in the terminal ileum are the cellular source of $\mathrm{p} 40$ protein leading to increased local levels of IL-23 (68). The IL-23/IL-17 axis has been implicated in several models of experimental colitis $(69,70)$. There is evidence that TLR-mediated induction of IL-23 is enhanced by nucleotide-binding oligomerization 2 (NOD2), resulting in the generation of DCs promoting the release of IL-17 by T cells (71). Of note, NOD2 mutations have been described as predisposing to Crohn disease in humans (72). Nonetheless, a recent study has shown that muramyl dipeptide (MDP) activation of NOD2 may have inhibitory effects by inhibiting cytokine responses of mouse DCs to various TLR ligands (73).

NOD2-mutant DCs derived from patients with Crohn disease failed to efficiently activate effector Th17 cells when stimulated with bacterial peptidoglycan or a combination of MDP and TLR ligands. These data suggest a pathway for IL-1- and IL-23-dependent priming of effector Th17 cells through NOD2-mediated detection of intracellular MDP, thus connecting 2 systems implicated in the pathogenesis of Crohn disease (74). The link between NOD2 and Th17 effector function warrants further exploration in order to ascertain the role of impaired innate immunity in Crohn disease.

Recent genome-wide association studies have linked the autophagy-related gene products autophagy-related 16-like 1 (ATG16L1) and immunity-related GTPase family, M (IRGM) with the pathogenesis of Crohn disease (75). Autophagy is a process involving the 


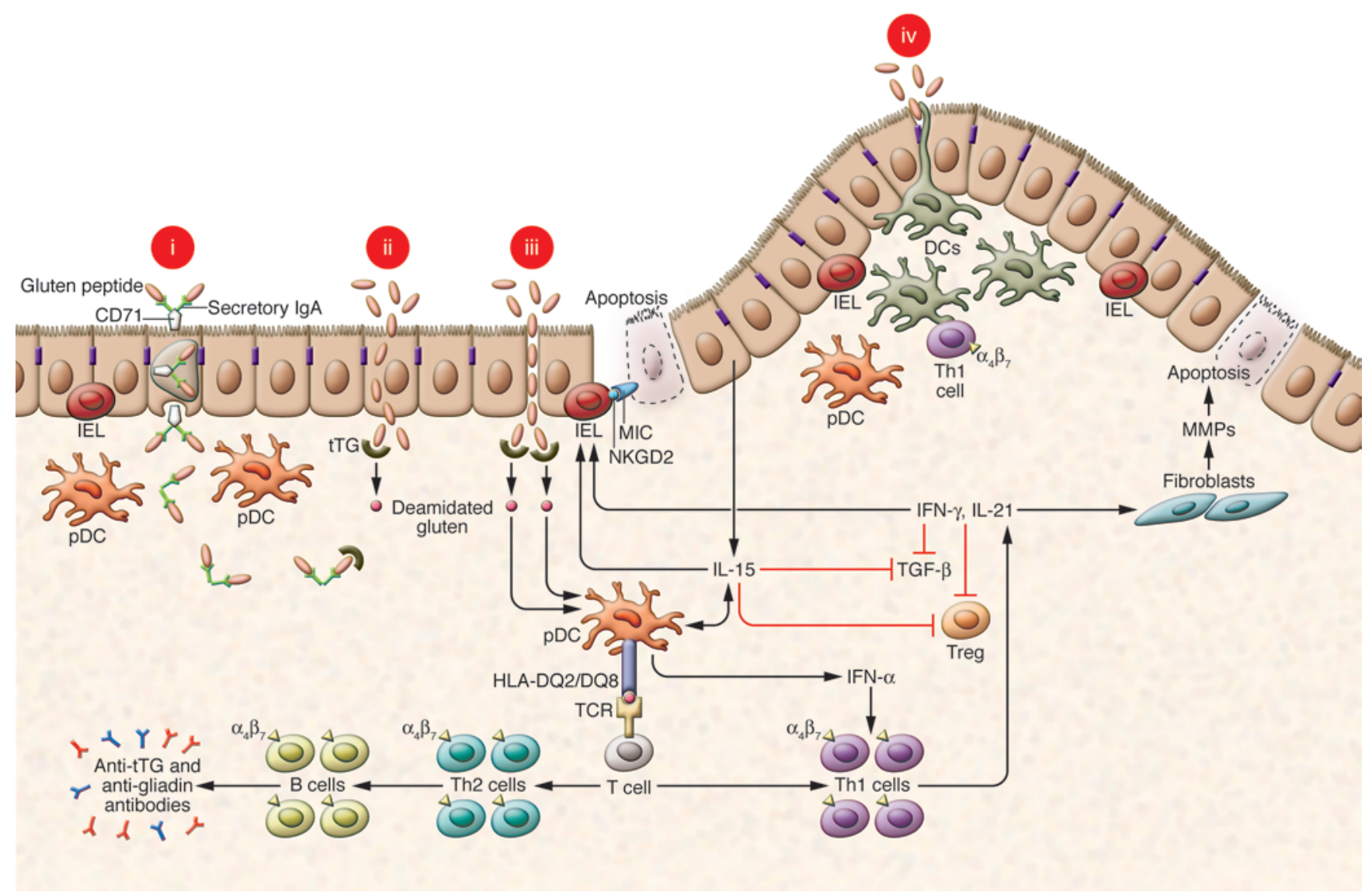

\section{Figure 3}

Possible role of DCs in celiac disease. Gluten peptides are transported across the intestinal epithelium via: retrotranscytosis (i), a protected retrotransport of secretory IgA via transferrin receptor CD71, which allows the entry of intact and thus harmful peptides into the intestinal mucosa; by a transcellular route (ii); or by a paracellular route (iii) as a consequence of impaired mucosal integrity. Gluten peptides might also be sampled by DCs extending their protrusions into the lumen (iv). Deamidation of gluten peptides by tissue transglutaminase (tTG) reinforces presentation of gluten peptides by pDCs to T cells in the context of HLA-DQ2 or HLA-DQ8 molecules. Activated, gluten-reactive Th1 cells produce high levels of proinflammatory cytokines (e.g., IFN- $\gamma$ and IL-21), which promote fibroblast secretion of MMPs responsible for degradation of ECM and basement membrane, and increase the intraepithelial lymphocyte (IEL) cytotoxicity through interaction between the homodimeric NK-activating receptor NFG2D and the MHC class I-related ligands (MIC), thus leading to epithelial cell apoptosis. IFN- $\alpha$ released by activated pDCs perpetuates the inflammatory reaction by inducing Th1 cells to produce IFN- $\gamma$. IL-21 and IL- 15 produced by DCs and IECs inhibit TGF- $\beta$ signaling and Treg function. Additionally, through the production of Th2 cytokines, Th2 cells drive the activation and clonal expansion of B cells, which differentiate into plasma cells producing anti-gliadin and anti-tissue transglutaminase antibodies. The latter have proven to be highly valuable in the diagnosis of celiac disease, as they are present in $98 \%$ of celiac patients on a gluten-containing diet.

degradation of captured proteins and cytoplasmic organelles via the formation of a double membrane structure that surrounds the cytoplasm. Recently, mice deficient in Atg16L1 have been generated (76). Macrophages isolated from these mice show an increase in IL-1 $\beta$ release in response to endotoxin (76). It is not known whether this defect is present also in DCs, but, if so, DCs may contribute to the increased inflammatory response observed in Crohn disease patients. Hence, given the crucial role of intestinal DCs in inducing either Tregs or Th17 cell development (characteristics associated with different subsets of DCs), it is likely that deregulation of the number or functions of these DC subsets - either intrinsic to the DCs or extrinsic because of lack of immunomodulatory signals provided by the local microenvironment - may result in imbalanced immune responses and disruption of gut homeostasis.

$D C \sin I B D$. DCs accumulate at sites of inflammation in patients with $\operatorname{IBD}(77,78)$, mainly as a consequence of upregulated muco- sal expression of chemokines such as CCL20 (79) or of addressins such as mucosal vascular addressin cell adhesion molecule-1 (MAdCAM-1; refs. 80, 81). DCs possess receptors for both of these gut-homing molecules: CCR6 and integrin $\alpha_{4} \beta_{7}$, respectively (82, 83). The observation of DC recruitment into the gut complements the evidence that pDCs and myeloid DCs are depleted in the peripheral blood of IBD patients with active disease (83).

In Crohn disease lesions, an increased number of CD83 ${ }^{+} \mathrm{LP}$ DCs is associated with numerous $\mathrm{CD}^{-} 3^{-} \mathrm{CD} 80^{+}$-specific intercellular adhesion molecule 3 -grabbing nonintegrin-positive $\left(\mathrm{DC}^{-S I G N}{ }^{+}\right)$ DCs producing IL-12 and IL-18 (84). The expression of TLR2, TLR4, and CD40 is enhanced in DCs isolated from inflamed mucosa (78). Furthermore, mature myeloid DCs, recruited as a consequence of the overexpression of lymphoid chemokines, form clusters with proliferating $\mathrm{T}$ cells in the affected colonic tissue (85). M-DC8 ${ }^{+}$cells, which have been recently identified as 
a new subpopulation of DCs in human blood expressing high levels of Fc $\gamma$ RIII (CD16) and secreting TNF- $\alpha$, are increased in the inflamed mucosa $(86,87)$, which indicates that DCs are an additional source of TNF- $\alpha$ in Crohn disease. Interestingly, antiTNF- $\alpha$ treatment induces a decrease of mucosal DC activation in Crohn disease patients (78).

In Crohn disease, activated DCs may migrate from the mucosa to the MLNs. At least 3 different myeloid DC populations are present in MLNs from Crohn disease patients: immature DCSIGN $^{+}$DCs in the medullary cords, DCs expressing the myeloid marker BDCA3 (CD141) around the lymph follicles, and mature $\mathrm{CD}^{2} 3^{+} \mathrm{DCs}$ expressing the S-100 protein (a marker for a subset of DCs named interdigitating reticulum cells) in the T cell areas (88). $\mathrm{CD}_{123}{ }^{+} \mathrm{pDCs}$ are rarely found in MLNs and colonic mucosa of both Crohn disease patients and healthy individuals $(20,88)$, while they appear to be predominant in normal duodenal mucosa (89). The functional significance of this variation in DC subsets along the bowel is currently under investigation.

It has been shown that a perturbation of the cross-talk between IECs and DCs may disrupt the intestinal immune homeostasis, thus promoting gut inflammation. Notably, IECs isolated from $70 \%$ of Crohn disease patients do not express TSLP and fail to control the DC-mediated proinflammatory response (21), resulting in an abnormal release of IL-12 by DCs, which drives Th1-type inflammatory responses $(21,90)$. There is also evidence that NOD2 mutations may affect the response of Crohn disease monocytederived DCs to bacteria (91), and DCs derived from NOD2-deficient Crohn disease patients show an impaired capacity to induce IL-17 expression upon MDP triggering (71).

Although the investigation of DCs in ulcerative colitis has received less attention, there is some evidence supporting their pathogenic role in this condition. Numerous basal aggregates formed by lymphocytes and $\mathrm{CD}^{+} 0^{+}$dendritiform cells resembling activated DCs are present in the colonic mucosa of patients affected by ulcerative colitis (92). Murakami et al. showed an increase of mucosal $\mathrm{CD}^{2} 3^{+}$and $\mathrm{CD} 6^{+}$cells producing macrophage inhibitory factor (93), which is thought to contribute to neutrophil recruitment and activation in ulcerative colitis. Additionally, DCs derived from peripheral monocytes of patients with ulcerative colitis are capable of increased immunostimulatory action (94).

It is known that DCs may be the source of IL-27, an IL-12-related cytokine that seems to be implicated in the pathogenesis of ulcerative colitis. Indeed, an increase in the expression of the IL-27 subunit EBI-3, which is produced by macrophages and DCs, has been observed in the LP of ulcerative colitis patients (95). Interestingly, EBI-3-deficient mice - having few NKT cells - are resistant to oxazolone-induced colitis (96), a mouse model of NKT-dependent ulcerative colitis (97). Together, these findings suggest that IL-27secreting DCs may be implicated in the pathogenesis of ulcerative colitis through the activation of NKT cells.

$D C$ in celiac disease. Celiac disease, the most common food-induced disease in the Western world with a prevalence close to 1 in 100 individuals, is a chronic inflammation of the small bowel induced in genetically susceptible individuals by the ingestion of proline- and glutamine-rich proteins contained in wheat (gliadins), rye (hordeins), and barley (secalins). The disease is characterized by an impressive clinical heterogeneity, ranging from totally asymptomatic to fully symptomatic forms manifesting with frank malabsorption; by increased morbidity as a result of frequent association with autoimmune disorders; and by increased mortality as a consequence of the emergence of $\mathrm{T}$ cell clonal proliferations predisposing to the development of an enteropathy-type T cell lymphoma (98).

Despite increasing evidence for the importance of DCs in maintaining the balance between immune activation and tolerance in the gut (99), our knowledge of the role played by DCs in celiac disease is still inadequate. Advances in the pathogenesis of celiac disease have focused on the mechanisms by which, after crossing the epithelium into the LP, gliadin peptides are deamidated by tissue transglutaminase and then presented by HLA-DQ2 ${ }^{+}$or HLA-DQ8 ${ }^{+}$ APCs to pathogenic $\mathrm{CD}^{+}{ }^{+} \mathrm{T}$ cells. The latter, once activated, drive a Th1 response leading to villous atrophy (98). DCs appear to exert at least 2 major functions in celiac disease. First, they are involved as APCs in the presentation of gliadin peptides to mucosal CD4 ${ }^{+}$ $T$ cells. Second, they may promote the persistence of the inflammatory response by interacting with LP T cells (Figure 3).

There are a number of critical questions relating to the role of DCs in celiac disease, and few studies have drawn comparisons between the phenotype and function of DCs isolated from normal and celiac small bowel. The number of myeloid DCs is elevated in active celiac mucosa compared with that in treated celiac mucosa and healthy controls, and the cells display an activated and mature phenotype $(89,100)$. Moreover, when CD $11 c^{+}$DCs isolated from untreated celiac mucosa are cultured in vitro with gliadin, they are more efficient than HLA-DQ2 ${ }^{+}$macrophages in activating gliadin-reactive T cells (100). However, there is an unexpected absence of $\mathrm{CD}_{123^{+}}$pDCs (100), which are known to exert a crucial role in inflammation by linking innate with adaptive immunity and eliciting Th1 polarization through IFN- $\alpha$ secretion (101). DCs are increased in untreated celiac mucosa, but the majority of the increase is reported to be caused by the pDC subset (89). This increase may reflect the depletion of $\mathrm{pDCs}$ found in the peripheral blood of untreated celiac patients (102), most likely occurring as a consequence of LP MAdCAM-1 overexpression (103). In active celiac mucosa, $\mathrm{CD} 123^{+}$DCs express higher levels of activation markers (i.e., CD80/CD86) and maturation markers (i.e., increased CD83), and sorted DCs contain higher transcript levels of IFN- $\alpha$ (89), a cytokine known to be overexpressed in celiac disease (104). Increased IL-23p19 transcripts are also observed in DCs from untreated celiac patients. Additionally, the decreased IL-10 and TGF- $\beta$ transcripts found in celiac DCs suggests that an additional way of breaking tolerance to gluten is via downregulation of antiinflammatory signals (89).

An intriguing aspect of the relationship between gluten and DCs was the observation that gluten and some gluten peptides directly induce maturation of both mouse $(105,106)$ and human DCs (107-110). The nonimmunodominant p31-43/49 fragment of $\alpha$-gliadin, presumed to be incapable of stimulating gluten-reactive $\mathrm{CD}^{+} \mathrm{T}$ cells, induced CD83 expression on CD3- LP cells in celiac biopsies grown ex vivo (107), thus emphasizing the involvement of the innate immune system in celiac disease. The argument for this involvement is further strengthened by the demonstration that a peptic digest of gliadin caused phenotypical and functional maturation of blood-derived immature DCs from human healthy individuals (108) and induced a higher maturation state in monocytes from untreated celiac patients than in treated celiac patients and healthy controls (109). Interestingly, gliadin enhanced the maturation of blood-derived immature DCs in all subjects irrespective of their genotype or of the presence of celiac disease, although gliadin-stimulated DCs from untreated celiac patients showed increased stimulation of autologous $\mathrm{T}$ cells compared with the 
other groups (110). Furthermore, gliadin plays a detrimental role in the regulation of NK cell-DC interactions, as it may switch the NK cell-DC cross-talk from a tolerogenic pathway (based on immature DC elimination) to a pathogenic one in which gliadin inhibits NK cell cytotoxicity against immature DCs via the CD94/ NKG2A pathway (111).

How gluten peptides enter the LP (whether paracellularly, as a consequence of tight junction opening, or transcellularly) remains unknown. On the basis of the abundant expression of surface tissue transglutaminase on celiac DCs, a role has been hypothesized for tissue transglutaminase-mediated endocytosis of gluten by DCs in contributing to the preferential recognition of deamidated gliadin peptides by $\mathrm{CD} 4^{+} \mathrm{T}$ cells $(112)$. However, the direct implication of this mechanism in the loading and subsequent generation of deamidated gluten peptides in celiac DCs is unclear.

\section{DCs as therapeutic targets}

The ability of DCs to induce tolerance has led to a number of studies using these cells therapeutically in order to control unwanted immune responses in models of allograft rejection, graft-versushost disease, and autoimmune disorders $(113,114)$. Different approaches have been designed to obtain the selective enhancement of tolerogenic properties of DCs, including biologic agents transfected into DCs or exposed to DCs in vitro; drugs that target DCs to induce and promote their tolerogenic properties; and immature or TNF- $\alpha$-induced semimature DCs. The aim of these strategies is to prevent the maturation of DCs and weaken their capacity to stimulate $\mathrm{T}$ cells (115).

Treatment with biological agents, such as IL-10, has been proposed in order to obtain stable tolerogenic DCs. IL-10-treated DCs actively downregulate costimulatory molecules, inhibit IFN- $\gamma$ secretion by activated $T$ cells, and reduce the production of antibodies by activated B cells (116). Among the various drugs implicated in the promotion of DC tolerogenicity, $1 \alpha, 25$-dihydroxivitamin $\mathrm{D}_{3}$, the active form of vitamin $\mathrm{D}_{3}$, induces the differentiation of tolerogenic DCs with upregulated expression of the inhibitory receptor immune globulin-like transcript 3, increased secretion of IL-10, and induction of Tregs (117). Mycophenolate has been shown to retard maturation of DCs and synergize with $1 \alpha, 25$-dihydroxivitamin $\mathrm{D}_{3}$ to promote the development of Tregs through an effect on DC maturation (118). Immunosuppressive or immunomodulatory agents, such as corticosteroids, estrogen, rapamycin, chloroquine, and tacrolimus, have the capacity to render DCs tolerogenic by inhibiting the differentiation and maturation of DCs, by reducing the production of costimulatory molecules and IL-12, and by inhibiting $\mathrm{T}$ cell costimulatory capacity (119). Interestingly, a common property of drugs targeting DCs is their capacity to inhibit NF- $\mathrm{\kappa}$, a signal transduction pathway known to play a crucial role in the inflammatory response. RelB, a member of the NF- $\mathrm{KB}$ family, is required for myeloid DC differentiation, and antigenpulsed DCs with inhibition of RelB function induce regulatory $\mathrm{CD}^{+} \mathrm{T}$ cells, which in turn may transfer tolerance to primed recipients in an IL-10-dependent fashion (120).

An intriguing approach to inducing tolerance in the gut comes from the evidence that IECs participate in the development of tolerogenic mucosal DCs. As mentioned above, epithelium-derived RA and TGF- $\beta$ are required to drive the development of CD $103^{+}$ tolerogenic DCs, which are potent inducers of Treg differentiation and suppression of Th1 responses (43). These findings could open new doors in the field of IBD therapeutics aimed at restoring the tolerogenic potential of resident DCs. In addition, the modification of DC function by in vivo or in vitro administration of probiotic bacteria has been proposed as a possible therapeutic strategy in IBD patients (121). Probiotics are live microorganisms similar to beneficial bacteria found in the human gut; when administered in adequate amounts, they confer a health benefit on the host. Interestingly, the effect of probiotics on DCs is dependent on TLR2 and NOD2 and the generation of Tregs (122).

DCs could be even more relevant to the therapy of celiac disease in which, unlike IBD, the antigen (gluten) is known. A potential DC-based, antigen-specific strategy could take advantage of the ability of DCs to expand and induce Tregs, which in turn suppress other DCs that present disease-producing antigens (123). In a spontaneous model of autoimmunity, the nonobese diabetic mouse, Tregs recognizing antigens in insulin-producing $\beta$ cells are generated by DCs and provide a therapeutic benefit (124). In the context of celiac disease, peptide vaccines developed by generating gliadin-loaded manipulated DCs could allow the formation of gliadin-specific Tregs, which in turn might educate DCs to initiate tolerance toward gluten. The identification of dominant epitopes to which all celiac patients respond represents a crucial step in the development of this approach, which will require the induction of tolerance to all $\mathrm{T}$ cell epitopes by vaccination with a few immunodominant epitopes. This strategy is expected to enter clinical trials soon (125). However, numerous aspects of this approach remain obscure. Peptide-loaded DCs may provide short-lived tolerance because of the dissociation rate of peptide binding. Moreover, it is not clear whether, in vivo, injected manipulated DCs have the ability to reach secondary lymphoid organs in order to favor the expansion of gliadin-specific Tregs and whether gliadin-specific Tregs have memory properties of long-term antigen-independent persistence.

\section{Conclusions}

In conclusion, deregulation of DC function, either as a primary effect of gene mutations or as a consequence of defective integration with environmental cues, may result in intestinal disease. Increased knowledge of DC function under healthy conditions and at the onset of disease may help our understanding not only of the precise role of DCs in the initiation and perpetuation of intestinal diseases, but also of their possible manipulation for therapeutic purposes.

Note added in proof. During publication of this review, a paper was published showing that blocking CD27-CD70 interaction can prevent experimental colitis (128). Another paper described the presence of tolerogenic CD123+ DCs in human MLNs (129).

\section{Acknowledgments}

M. Rescigno is supported by the $7^{\text {th }}$ framework Programme of the European Commission (METAhit, ERC, and IBDase), the Crohn's and Colitis Foundation of America (CCFA), the Italian Ministry of Health, the International Association for Cancer Research (AICR), and the Associazione Italiana per la Ricerca sul Cancro (AIRC).

Address correspondence to: Maria Rescigno, Department of Experimental Oncology, European Institute of Oncology, Via Ripamonti 435, 20141 Milan, Italy. Phone: 39-0257489925; Fax: 39-0257489871; E-mail: maria.rescigno@ifom-ieo-campus.it. 
1. Banchereau, J., and Steinman, R.M. 1998. Dendritic cells and the control of immunity. Nature. 392:245-252.

2. Steinman, R.M. 2007. Dendritic cells: understanding immunogenicity. Eur. J. Immunol. 37(Suppl. 1):S53-S60.

3. Mowat, A.M. 2003. Anatomical basis of tolerance and immunity to intestinal antigens. Nat. Rev. Immunol. 3:331-341.

4. Ueno, H., et al. 2007. Dendritic cell subsets in health and disease. Immunol. Rev. 219:118-142.

5. Huang, F.P., et al. 2000. A discrete subpopulation of dendritic cells transports apoptotic intestinal epithelial cells to $\mathrm{T}$ cell areas of mesenteric lymph nodes. J. Exp. Med. 191:435-444.

6. Coombes, J.L., and Powrie, F. 2008. Dendritic cells in intestinal immune regulation. Nat. Rev. Immunol. 8:435-446.

7. Dubin, P.J., and Kolls, J.K. 2008. Th17 cytokines and mucosal immunity. Immunol. Rev. 226:160-171.

8. Steinman, L. 2008. A rush to judgment on Th17. J. Exp. Med. 205:1517-1522.

9. Powrie, F., Read, S., Mottet, C., Uhlig, H., and Maloy, K. 2003. Control of immune pathology by regulatory T cells. Novartis Found. Symp. 252:92-98; discussion 98-105, 106-114.

10. Rescigno, M., et al. 2001. Dendritic cells express tight junction proteins and penetrate gut epithelial monolayers to sample bacteria. Nat. Immunol. 2:361-367.

11. Cerutti, A. 2008. The regulation of IgA class switching. Nat. Rev. Immunol. 8:421-434.

12. Kelsall, B. 2008. Recent progress in understanding the phenotype and function of intestinal dendritic cells and macrophages. Mucosal Immunol. 1:460-469.

13. Iwasaki, A. 2007. Mucosal dendritic cells. Annu. Rev. Immunol. 25:381-418.

14. Sun, C.M., et al. 2007. Small intestine lamina propria dendritic cells promote de novo generation of Foxp3 T reg cells via retinoic acid. J. Exp. Med. 204:1775-1785.

15. Atarashi, K., et al. 2008. ATP drives lamina propria $\mathrm{T}(\mathrm{H}) 17$ cell differentiation. Nature. 455:808-812.

16. Annacker, O., et al. 2005. Essential role for CD103 in the $\mathrm{T}$ cell-mediated regulation of experimental colitis. J. Exp. Med. 202:1051-1061.

17. Coombes, J.L., et al. 2007. A functionally specialized population of mucosal CD103+ DCs induces Foxp3+ regulatory T cells via a TGF- $\{$ beta $\}$ and retinoic acid dependent mechanism. J. Exp. Med. 204:1757-1764.

18. Johansson-Lindbom, B., et al. 2005. Functional specialization of gut CD103+ dendritic cells in the regulation of tissue-selective $\mathrm{T}$ cell homing. J. Exp. Med. 202:1063-1073.

19. Jaensson, E., et al. 2008. Small intestinal CD103+ dendritic cells display unique functional properties that are conserved between mice and humans. J. Exp. Med. 205:2139-2149.

20. Bell, S.J., et al. 2001. Migration and maturation of human colonic dendritic cells. J. Immunol. 166:4958-4967.

21. Rimoldi, M., et al. 2005. Intestinal immune homeostasis is regulated by the crosstalk between epithelial cells and dendritic cells. Nat. Immunol. 6:507-514.

22. Mucida, D., et al. 2007. Reciprocal TH17 and regulatory $\mathrm{T}$ cell differentiation mediated by retinoic acid. Science. 317:256-260.

23. Di Sabatino, A., et al. 2008. Blockade of transforming growth factor beta upregulates T-box transcription factor T-bet, and increases T helper cell type 1 cytokine and matrix metalloproteinase- 3 production in the human gut mucosa. Gut. 57:605-612.

24. Uematsu, S., et al. 2008. Regulation of humoral and cellular gut immunity by lamina propria dendritic cells expressing Toll-like receptor 5. Nat. Immunol. 9:769-776.

25. Denning, T.L., Wang, Y.C., Patel, S.R., Williams, I.R., and Pulendran, B. 2007. Lamina propria mac- rophages and dendritic cells differentially induce regulatory and interleukin 17-producing $\mathrm{T}$ cell responses. Nat. Immunol. 8:1086-1094.

26. Niess, J.H., et al. 2005. CX3CR1-Mediated Dendritic Cell Access to the Intestinal Lumen and Bacterial Clearance. Science. 307:254-258.

27. Milling, S.W., et al. 2009. Steady-state migrating intestinal dendritic cells induce potent inflammatory responses in naive CD4+ T cells. Mucosal Immunol. 2:156-165.

28. Ishii, K.J., Koyama, S., Nakagawa, A., Coban, C., and Akira, S. 2008. Host innate immune receptors and beyond: making sense of microbial infections. Cell Host Microbe. 3:352-363.

29. Monteleone, I., Platt, A.M., Jaensson, E., Agace, W.W., and Mowat, A.M. 2008. IL-10-dependent partial refractoriness to Toll-like receptor stimulation modulates gut mucosal dendritic cell function. Eur. J. Immunol. 38:1533-1547.

30. Niess, J.H., Leithauser, F., Adler, G., and Reimann, J. 2008. Commensal gut flora drives the expansion of proinflammatory CD4 T cells in the colonic lamina propria under normal and inflammatory conditions. J. Immunol. 180:559-568.

31. Zaph, C., et al. 2008. Commensal-dependent expression of IL-25 regulates the IL-23-IL-17 axis in the intestine. J. Exp. Med. 205:2191-2198.

32. Laouar, A., et al. 2005. CD70+ antigen-presenting cells control the proliferation and differentiation of $\mathrm{T}$ cells in the intestinal mucosa. Nat. Immunol. 6:698-706.

33. Iwasaki, A., and Kelsall, B.L. 2001. Unique functions of cd11b(+), cd8alpha (+), and double-negative peyer's patch dendritic cells. J. Immunol. 166:4884-4890.

34. Sato, A., et al. 2003. CD11b+ Peyer's patch dendritic cells secrete IL- 6 and induce IgA secretion from naive B cells. J. Immunol. 171:3684-3690.

35. Mora, J.R., et al. 2006. Generation of Gut-Homing IgA-Secreting B Cells by Intestinal Dendritic Cells. Science. 314:1157-1160.

36. Tezuka, H., et al. 2007. Regulation of IgA production by naturally occurring TNF/iNOS-producing dendritic cells. Nature. 448:929-933.

37. Travis, M.A., et al. 2007. Loss of integrin alpha(v) beta 8 on dendritic cells causes autoimmunity and colitis in mice. Nature. 449:361-365.

38. Fagarasan, S., Kinoshita, K., Muramatsu, M., Ikuta, K., and Honjo, T. 2001. In situ class switching and differentiation to IgA-producing cells in the gut lamina propria. Nature. 413:639-643.

39. Tsuji, M., et al. 2008. Requirement for lymphoid tissue-inducer cells in isolated follicle formation and $\mathrm{T}$ cell-independent immunoglobulin A generation in the gut. Immunity. 29:261-271.

40. Varol, C., et al. 2007. Monocytes give rise to mucosal, but not splenic, conventional dendritic cells. J. Exp. Med. 204:171-180.

41. Iliev, I.D., Matteoli, G., and Rescigno, M. 2007. The yin and yang of intestinal epithelial cells in controlling dendritic cell function. J. Exp. Med. 204:2253-2257.

42. Zaph, C., et al. 2007. Epithelial-cell-intrinsic IKKbeta expression regulates intestinal immune homeostasis. Nature. 446:552-556

43. Iliev, I.D., Mileti, E., Matteoli, G., Chieppa, M., and Rescigno, M. 2009. Intestinal epithelial cells promote colitis-protective regulatory T-cell differentiation through dendritic cell conditioning. Mucosal Immunol. 2:340-350.

44. Xu, W., et al. 2007. Epithelial cells trigger frontline immunoglobulin class switching through a pathway regulated by the inhibitor SLPI. Nat. Immunol. 8:294-303.

45. He, B., et al. 2007. Intestinal bacteria trigger T cellindependent immunoglobulin A(2) class switching by inducing epithelial-cell secretion of the cytokine APRIL. Immunity. 26:812-826.

46. Cerutti, A., and Rescigno, M. 2008. The biology of intestinal immunoglobulin A responses. Immunity. 28:740-750.

47. Zeuthen, L.H., Fink, L.N., and Frokiaer, H. 2008. Epithelial cells prime the immune response to an array of gut-derived commensals towards a tolerogenic phenotype through distinct actions of thymic stromal lymphopoietin and transforming growth factor-beta. Immunology. 123:197-208.

48. Smythies, L.E., et al. 2005. Human intestinal macrophages display profound inflammatory anergy despite avid phagocytic and bacteriocidal activity. J. Clin. Invest. 115:66-75.

49. Zhang, M., et al. 2004. Splenic stroma drives mature dendritic cells to differentiate into regulatory dendritic cells. Nat. Immunol. 5:1124-1133.

50. Svensson, M., Maroof, A., Ato, M., and Kaye, P.M. 2004. Stromal cells direct local differentiation of regulatory dendritic cells. Immunity. 21:805-816.

51. Newberry, R.D., McDonough, J.S., Stenson, W.F., and Lorenz, R.G. 2001. Spontaneous and continuous cyclooxygenase-2-dependent prostaglandin E2 production by stromal cells in the murine small intestine lamina propria: directing the tone of the intestinal immune response. J. Immunol. 166:4465-4472.

52. Braun, D., Longman, R.S., and Albert, M.L. 2005. A two-step induction of indoleamine 2,3 dioxygenase (IDO) activity during dendritic-cell maturation. Blood. 106:2375-2381.

53. Contractor, N., Louten, J., Kim, L., Biron, C.A., and Kelsall, B.L. 2007. Cutting edge: Peyer's patch plasmacytoid dendritic cells (pDCs) produce low levels of type I interferons: possible role for IL-10, TGFbeta, and prostaglandin E2 in conditioning a unique mucosal pDC phenotype. J. Immunol. 179:2690-2694

54. Yokota, A., et al. 2009. GM-CSF and IL-4 synergistically trigger dendritic cells to acquire retinoic acidproducing capacity. Int. Immunol. 21:361-377.

55. Tiemessen, M.M., et al. 2007. CD4+CD25+Foxp3+ regulatory $\mathrm{T}$ cells induce alternative activation of human monocytes/macrophages. Proc. Natl. Acad. Sci. U. S. A. 104:19446-19451.

56. Loftus, E.V., Jr. 2004. Clinical epidemiology of inflammatory bowel disease: Incidence, prevalence, and environmental influences. Gastroenterology. 126:1504-1517.

57. Baumgart, D.C., and Sandborn, W.J. 2007. Inflammatory bowel disease: clinical aspects and established and evolving therapies. Lancet. 369:1641-1657.

58. Macdonald, T.T., and Monteleone, G. 2005. Immunity, inflammation, and allergy in the gut. Science. 307:1920-1925.

59. Malmstrom, V., et al. 2001. CD134L expression on dendritic cells in the mesenteric lymph nodes drives colitis in T cell-restored SCID mice. J. Immunol. 166:6972-6981.

60. Leithauser, F., Trobonjaca, Z., Moller, P., and Reimann, J. 2001. Clustering of colonic lamina propria CD4(+) T cells to subepithelial dendritic cell aggregates precedes the development of colitis in a murine adoptive transfer model. Lab. Invest. 81:1339-1349.

61. Uhlig, H.H., et al. 2006. Differential activity of IL-12 and IL-23 in mucosal and systemic innate immune pathology. Immunity. 25:309-318.

62. Abe, K., et al. 2007. Conventional dendritic cells regulate the outcome of colonic inflammation independently of T cells. Proc. Natl. Acad. Sci. U. S. A. 104:17022-17027.

63. Qualls, J.E., Tuna, H., Kaplan, A.M., and Cohen, D.A. 2009. Suppression of experimental colitis in mice by CD $11 \mathrm{c}^{+}$dendritic cells. Inflamm. Bowel Dis. 15:236-247.

64. Mottet, C., Uhlig, H.H., and Powrie, F. 2003. Cutting edge: cure of colitis by CD4+CD $25+$ regulatory T cells. J. Immunol. 170:3939-3943.

65. Westendorf, A.M., et al. 2009. CD4+Foxp3+ regulatory $\mathrm{T}$ cell expansion induced by antigen-driven interaction with intestinal epithelial cells indepen- 
dent of local dendritic cells. Gut. 58:211-219.

66. Krajina, T., Leithauser, F., Moller, P., Trobonjaca, Z., and Reimann, J. 2003. Colonic lamina propria dendritic cells in mice with CD4+ T cell-induced colitis. Eur. J. Immunol. 33:1073-1083.

67. Korn, T., Bettelli, E., Oukka, M., and Kuchroo, V.K. 2009. IL-17 and Th17 cells. Annu. Rev. Immunol. 27:485-517.

68. Becker, C., et al. 2003. Constitutive p40 promoter activation and IL-23 production in the terminal ileum mediated by dendritic cells. J. Clin. Invest. 112:693-706.

69. Yen, D., et al. 2006. IL-23 is essential for T cellmediated colitis and promotes inflammation via IL-17 and IL-6. J. Clin. Invest. 116:1310-1316.

70. Hue, S., et al. 2006. Interleukin-23 drives innate and $\mathrm{T}$ cell-mediated intestinal inflammation. J. Exp. Med. 203:2473-2483.

71. van Beelen, A.J., et al. 2007. Stimulation of the intracellular bacterial sensor NOD2 programs dendritic cells to promote interleukin-17 production in human memory T cells. Immunity. 27:660-669.

72. Rescigno, M., and Nieuwenhuis, E.E. 2007. The role of altered microbial signaling via mutant NODs in intestinal inflammation. Curr. Opin. Gastroenterol. 23:21-26.

73. Watanabe, T., et al. 2008. Muramyl dipeptide activation of nucleotide-binding oligomerization domain 2 protects mice from experimental colitis. J. Clin. Invest. 118:545-559.

74. Stetson, D.B., and Medzhitov, R. 2007. T helper 17 cells get the NOD. Immunity. 27:546-548.

75. Hampe, J., et al. 2007. A genome-wide association scan of nonsynonymous SNPs identifies a susceptibility variant for Crohn disease in ATG16L1. Nat. Genet. 39:207-211.

76. Saitoh, T., et al. 2008. Loss of the autophagy protein Atg16L1 enhances endotoxin-induced IL1 beta production. Nature. 456:264-268.

77. Vuckovic, S., et al. 2001. CD40 and CD86 upregulation with divergent CMRF44 expression on blood dendritic cells in inflammatory bowel diseases. Am. J. Gastroenterol. 96:2946-2956.

78. Hart, A.L., et al. 2005. Characteristics of intestinal dendritic cells in inflammatory bowel diseases. Gastroenterology. 129:50-65.

79. Kaser, A., et al. 2004. Increased expression of CCL20 in human inflammatory bowel disease. J. Clin. Immunol. 24:74-85.

80. Souza, H.S., Elia, C.C., Spencer, J., and MacDonald, T.T. 1999. Expression of lymphocyte-endothelial receptor-ligand pairs, alpha4beta7/MAdCAM-1 and OX40/OX40 ligand in the colon and jejunum of patients with inflammatory bowel disease. Gut. 45.856-863.

81. Arihiro, S., et al. 2002. Differential expression of mucosal addressin cell adhesion molecule- 1 (MAdCAM-1) in ulcerative colitis and Crohn's disease. Pathol. Int. 52:367-374.

82. Dieu, M.C., et al. 1998. Selective recruitment of immature and mature dendritic cells by distinct chemokines expressed in different anatomic sites. J. Exp. Med. 188:373-386.

83. Baumgart, D.C., et al. 2005. Patients with active inflammatory bowel disease lack immature peripheral blood plasmacytoid and myeloid dendritic cells. Gut. 54:228-236.

84. te Velde, A.A., et al. 2003. Increased expression of DCSIGN+IL-12+IL-18+ and CD83+IL-12-IL-18- dendritic cell populations in the colonic mucosa of patients with Crohn's disease. Eur. I. Immunol. 33:143-151.

85. Middel, P., Raddatz, D., Gunawan, B., Haller, F., and Radzun, H.J. 2006. Increased number of mature dendritic cells in Crohn's disease: evidence for a chemokine mediated retention mechanism. Gut. 55:220-227.

86. de Baey, A., Mende, I., Riethmueller, G., and Baeuerle, P.A. 2001. Phenotype and function of human dendritic cells derived from M-DC8(+) monocytes. Eur. J. Immunol. 31:1646-1655.

87. de Baey, A., et al. 2003. A subset of human dendritic cells in the $\mathrm{T}$ cell area of mucosa-associated lymphoid tissue with a high potential to produce TNF-alpha. J. Immunol. 170:5089-5094.

88. Verstege, M.I., et al. 2008. Dendritic cell populations in colon and mesenteric lymph nodes of patients with Crohn's disease. J. Histochem. Cytochem. 56:233-241.

89. Di Sabatino, A., et al. 2007. Evidence for the role of interferon-alfa production by dendritic cells in the Th1 response in celiac disease. Gastroenterology. 133:1175-1187.

90. Rimoldi, M, et al. 2005. Monocyte-derived dendritic cells activated by bacteria or by bacteria-stimulated epithelial cells are functionally different. Blood. 106:2818-2826.

91. Salucci, V., et al. 2008. Monocyte-derived dendritic cells from Crohn patients show differential NOD2/CARD15-dependent immune responses to bacteria. Inflamm. Bowel Dis. 14:812-818.

92. Yeung, M.M., et al. 2000. Characterisation of mucosal lymphoid aggregates in ulcerative colitis: immune cell phenotype and TcR-gammadelta expression. Gut. 47:215-227.

93. Murakami, H., Akbar, S.M., Matsui, H., Horiike, N., and Onji, M. 2002. Macrophage migration inhibitory factor activates antigen-presenting dendritic cells and induces inflammatory cytokines in ulcerative colitis. Clin. Exp. Immunol. 128:504-510.

94. Ikeda, Y., Akbar, F., Matsui, H., and Onji, M. 2001. Characterization of antigen-presenting dendritic cells in the peripheral blood and colonic mucosa of patients with ulcerative colitis. Eur. J. Gastroenterol. Hepatol. 13:841-850.

95. Christ, A.D., et al. 1998. An interleukin 12-related cytokine is up-regulated in ulcerative colitis but not in Crohn's disease. Gastroenterology. 115:307-313.

96. Nieuwenhuis, E.E., et al. 2002. Disruption of $\mathrm{T}$ helper 2-immune responses in Epstein-Barr virus-induced gene 3-deficient mice. Proc. Natl. Acad. Sci. U. S. A. 99:16951-16956.

97. Heller, F., Fuss, I.J., Nieuwenhuis, E.E., Blumberg, R.S. and Strober, W. 2002. Oxazolone colitis, a Th2 colitis model resembling ulcerative colitis, is mediated by IL-13-producing NK-T cells. Immunity. 17:629-638.

98. Di Sabatino, A., and Corazza, G.R. 2009. Coeliac disease. Lancet. 373:1480-1493.

99. Bilsborough, J., and Viney, J.L. 2004. Gastrointestinal dendritic cells play a role in immunity, tolerance, and disease. Gastroenterology. 127:300-309.

100.Raki, M., et al. 2006. A unique dendritic cell subset accumulates in the celiac lesion and efficiently activates gluten-reactive T cells. Gastroenterology. 131:428-438

101.Colonna, M., Trinchieri, G., and Liu, Y.J. 2004. Plasmacytoid dendritic cells in immunity. Nat. Immunol. 5:1219-1226.

102.Ciccocioppo, R., et al. 2007. Reduced number and function of peripheral dendritic cells in coeliac disease. Clin. Exp. Immunol. 149:487-496.

103. Di Sabatino, A., et al. 2009. Increased expression of mucosal addressin cell adhesion molecule 1 in the duodenum of patients with active celiac disease is associated with depletion of integrin alpha4beta7positive T cells in blood. Hum. Pathol. 40:699-704.

104.Monteleone, G., Pender, S.L., Wathen, N.C., and MacDonald, T.T. 2001. Interferon-alpha drives $\mathrm{T}$ cell-mediated immunopathology in the intestine. Eur. I. Immunol. 31:2247-2255.

105.Nikulina, M., Habich, C., Flohe, S.B., Scott, F.W., and Kolb, H. 2004. Wheat gluten causes dendritic cell maturation and chemokine secretion. J. Immu nol. 173:1925-1933.

106. Ciccocioppo, R., et al. 2008. Effects of gliadin stimulation on bone marrow-derived dendritic cells from HLA-DQ8 transgenic MICE. Dig. Liver Dis.
40:927-935.

107. Maiuri, L., et al. 2003. Association between innate response to gliadin and activation of pathogenic $\mathrm{T}$ cells in coeliac disease. Lancet. 362:30-37.

108. Palova-Jelinkova, L., et al. 2005. Gliadin fragments induce phenotypic and functional maturation of human dendritic cells. J. Immunol. 175:7038-7045.

109. Cinova, J., et al. 2007. Gliadin peptides activate blood monocytes from patients with celiac disease. J. Clin. Immunol. 27:201-209.

110. Rakhimova, M., et al. 2009. In vitro differentiation of human monocytes into dendritic cells by peptictryptic digest of gliadin is independent of genetic predisposition and the presence of celiac disease. J. Clin. Immunol. 29:29-37.

111. Terrazzano, G., et al. 2007. Gliadin regulates the NK-dendritic cell cross-talk by HLA-E surface stabilization. J. Immunol. 179:372-381.

112. Raki, M., et al. 2007. Surface expression of transglutaminase 2 by dendritic cells and its potential role for uptake and presentation of gluten peptides to T cells. Scand. J. Immunol. 65:213-220.

113. van Duivenvoorde, L.M., van Mierlo, G.J., Boonman, Z.F., and Toes, R.E. 2006. Dendritic cells: vehicles for tolerance induction and prevention of autoimmune diseases. Immunobiology. 211:627-632.

114.Steinman, R.M., and Banchereau, J. 2007. Taking dendritic cells into medicine. Nature. 449:419-426.

115.Xiao, B.G., Huang, Y.M., and Link, H. 2006. Tolerogenic dendritic cells: the ins and outs of outcome. J. Immunother. 29:465-471.

116. Corinti, S., Albanesi, C., la Sala, A., Pastore, S., and Girolomoni, G. 2001. Regulatory activity of autocrine IL-10 on dendritic cell functions. J. Immunol. 166:4312-4318

117.Penna, G., and Adorini, L. 2000. 1 Alpha,25-dihydroxyvitamin D3 inhibits differentiation, maturation, activation, and survival of dendritic cells leading to impaired alloreactive $\mathrm{T}$ cell activation. J. Immunol. 164:2405-2411.

118. Mehling, A., et al. 2000. Mycophenolate mofetil impairs the maturation and function of murine dendritic cells. J. Immunol. 165:2374-2381.

119. Adorini, L., Giarratana, N., and Penna, G. 2004. Pharmacological induction of tolerogenic dendritic cells and regulatory T cells. Semin. Immunol. 16:127-134.

120.Martin, E., O'Sullivan, B., Low, P., and Thomas, R. 2003. Antigen-specific suppression of a primed immune response by dendritic cells mediated by regulatory $\mathrm{T}$ cells secreting interleukin-10. Immunity. 18:155-167.

121.Boirivant, M., and Strober, W. 2007. The mechanism of action of probiotics. Curr. Opin. Gastroenterol. 23:679-692.

122. Foligne, B., et al. 2007. A key role of dendritic cells in probiotic functionality. PLOS ONE. 2:e313.

123. Steinman, R.M. 2008. Dendritic cells in vivo: a key target for a new vaccine science. Immunity. 29:319-324.

124. Tarbell, K.V., et al. 2007. Dendritic cell-expanded, islet-specific CD4+CD25+CD62L+ regulatory $\mathrm{T}$ cells restore normoglycemia in diabetic NOD mice. J. Exp. Med. 204:191-201.

125. Sollid, L.M., and Lundin, K.E. 2009. Diagnosis and treatment of celiac disease. Mucosal Immunol. 2:3-7.

126.Salazar-Gonzalez, R.M., et al. 2006. CCR6-mediated dendritic cell activation of pathogen-specific $\mathrm{T}$ cells in Peyer's patches. Immunity. 24:623-632.

127. Broere, F., et al. 2009. Cyclooxygenase-2 in mucosal DC mediates induction of regulatory $\mathrm{T}$ cells in the intestine through suppression of IL-4. Mucosal Immunol. 2:254-264.

128. Manocha, M., et al. 2009. Blocking CD27-CD70 costimulatory pathway suppresses experimental colitis. J. Immunol. 183:270-276.

129.Iliev, I.D., et al. 2009. Human intestinal epithelial cells promote the differentiation of tolerogenic dendritic cells. Gut. Online publication ahead of print. doi: 10.1136/gut.2008.175166. 